\title{
CONDICIONES INSTITUCIONALES DE HIGIENE, SALUD, SEGURIDAD Y MEDIO AMBIENTE, EN LA POBLACIÓN ESTUDIANTIL DE INSTITUCIONES eduCATivas téCNiCas EN Pasto
}

\author{
Fabio Andrés Bolaños Alomia ${ }^{1}$
}

${ }^{1}$ Docente de tiempo completo de Ingeniería Industrial, Universidad Cooperativa de Colombia, Pasto, Colombia Correo electrónico: fabio.bolanos@campusucc.edu.co

Recibido: 5 de marzo del 2014. Aprobado: 10 de junio del 2013.

Cómo citar este artículo: F. A. Bolaños-Alomia, "Condiciones institucionales de higiene, salud, seguridad y medio ambiente, en la población estudiantil de instituciones educativas técnicas en Pasto". Ingeniería Solidaria, vol. 10, n. ${ }^{\circ}$ 17, pp. 93-103, en.-dic., 2014. doi: http://dx.doi.org/10.16925/in.v9i17.809

\begin{abstract}
Resumen. En esta investigación se presenta el resultado del diagnóstico de las condiciones institucionales de higiene, salud, seguridad y medio ambiente, en las instituciones Luis Delfín Insuasty Rodríguez (INEM) e Instituto Técnico Superior Industrial Municipal (ITSIM) en el 2012; se identificaron los factores de riesgo asociados a las condiciones mencionadas, también se identificaron las actividades de prevención y promoción que deben llevarse a cabo en dichas instituciones para minimizar el impacto que los riesgos identificados puedan causar sobre la población de las instituciones educativas, lo anterior teniendo en cuenta la normatividad vigente en el país en términos de la salud ocupacional. Los resultados se obtuvieron después de realizar una encuesta, unas entrevistas semiestructuradas y el levantamiento de los panoramas de factores de riesgo de cada institución, de acuerdo con la norma vigente colombiana GTC 45 para la elaboración de panoramas de factores de riesgo.
\end{abstract}

Palabras clave: salud ocupacional, seguridad, higiene, medio ambiente, panorama de factores de riesgo.

Institutional Hygiene, Health, SAFETy AND THE ENVIRONMENTAL CONDITIONS among a Student Population at Technical Educational Institutions in Pasto

Abstract. This research presents the results from a diagnosis of institutional hygiene, health, safety and environmental conditions at the Luis Delfín Insuasty Rodríguez (INEM) and Instituto Técnico Superior Industrial Municipal (ITSIM) educational institutions in 2012; risk factors associated with the above-mentioned conditions were identified, along with activities for prevention and promotion that must be undertaken to minimize possible impacts from the identified risks among the population at the educational institutions, taking into account currently-ineffect occupational health regulations in the country. The results were obtained after carrying out a survey, semi-structured interviews and risk factor assessments at each institution, pursuant to currently-in-effect Colombian standard GTC 45 for such risk factor assessments.

Keywords: occupational health, safety, hygiene, the environment, risk factor assessment.
CONDIÇõES INSTITUCIONAIS DE HIGIENE, SAÚDE, SEGURANÇA E MEIO AMBIENTE NA POPULAÇÃO ESTUDANTIL DE INSTituições educativas técnicas em Pasto

Resumo. Nesta pesquisa, apresenta-se o resultado do diagnóstico das condições institucionais de higiene, saúde, segurança e meio ambiente nas instituições Luis Delfín Insuasty Rodríguez (INEM) e Instituto Técnico Superior Industrial Municipal (ITSIM) em 2012; identificaram-se os fatores de risco associados às condições mencionadas, também se identificaram as atividades de prevenção e promoção que devem ser realizadas nessas instituições para minimizar o impacto que os riscos identificados possam causar na população das instituições educativas, considerando a normatividade vigente no país em termos de saúde ocupacional. Os resultados foram obtidos depois de realizar uma enquete, entrevistas semiestruturadas e levantamento dos panoramas de fatores de risco de cada instituição, de acordo com a norma vigente colombiana GTC 45 para a elaboração de panoramas de fatores de risco.

Palavras-chave: saúde ocupacional, segurança, higiene, meio ambiente, panoramas de fatores de risco. 


\section{Introducción}

Las instituciones académicas consideradas como empresas de servicios, constantemente buscan elevar el nivel académico. Esto a raíz de que las leyes y normas actuales exigen actividades y programas que buscan garantizar altos niveles de calidad académica, a través, por ejemplo, del diseño e implementación de planes de Salud Ocupacional, tal como lo estipula la norma técnica colombiana GTC 34 en la cual se establece lo pertinente para la elaboración e implementación de un programa de salud ocupacional, Proyectos Educativos, y Sistemas de Gestión de Calidad. De esta manera, las instituciones pretenden avanzar en su nivel de competitividad para permanecer en el tiempo y adquirir prestigio. A pesar de todas estas actividades, en muchas ocasiones el estudio y desarrollo de estos planes se enfocan en la calidad de enseñanza, basadas en mejoras del conocimiento y las condiciones administrativas, dejando en un segundo plano los aspectos relacionados con salud y bienestar de la población estudiantil, la cual posee una extensa participación en la mayoría de regiones de Colombia con un total de 8700000 estudiantes [1] en los niveles de educación básica y media.

Muchas instituciones educativas ofrecen, además de la modalidad académica, otras opciones de formación en el nivel de educación media que se tuvieron en cuenta en este estudio como las técnico industriales, las cuales, para garantizar un correcto proceso de enseñanza-aprendizaje, requieren el uso y manipulación de maquinaria, herramientas y equipo que pueden llegar a causar incidentes o accidentes.

En la población estudiantil (en sus modalidades académica y técnica industrial) se presentan variedad de problemas relacionados con su bienestar, tales como el deterioro de la salud, exposiciones a riesgos ocupacionales, falta de cuidado de la seguridad, problemas de higiene y mala relación con el medio ambiente, los cuales interfieren directamente con el desarrollo normal de las actividades académicas y afectan a los estudiantes en sus diferentes ámbitos: personal, institucional, familiar y social. Por lo tanto, es necesario tener en cuenta e involucrar a la población estudiantil en la formulación y desarrollo de planes y proyectos que busquen la calidad. Para ello, es importante hacer un diagnóstico de las condiciones actuales y reales en las que se encuentran los estudiantes, con el fin de determinar acciones preventivas y correctivas encaminadas a mitigar las consecuencias de los riesgos a los que se ven sometidos los estudiantes y en general toda la comunidad académica de las instituciones objeto de estudio.

\section{Material y métodos}

La investigación realizada es de tipo descriptivo, puesto que se enfocó hacia el conocimiento de las condiciones institucionales de las instituciones educativas técnicas objeto de estudio en cuanto al estado de salud, seguridad, higiene y medio ambiente.

En la investigación se observaron los planteles y el ambiente de estudio con el objeto de indagar desde el punto de vista de los estudiantes el estado actual de sus instituciones y el cuidado que se tiene con ellos, se realizó una encuesta a la población seleccionada mediante muestreo, a 127 en el ITSIM y 218 en el INEM para un total de 345 encuestas, Para obtener el tamaño de la muestra [2] se utilizó el método de muestreo probabilístico aleatorio simple [3] con un margen de error de $5 \%$ y nivel de confianza de $95 \%$.

Los grados que se tuvieron en cuenta fueron octa$\operatorname{vos}\left(8^{\circ}\right)$, novenos $\left(9^{\circ}\right)$, decimos $\left(10^{\circ}\right)$ y onceavos $\left(11^{\circ}\right)$ matriculados en el periodo B del año 2012 de las Instituciones Luis Delfín Insuasty Rodríguez (INEM) [4] e Instituto Técnico Superior Industrial Municipal (ITSIM) [5] de Pasto, que son las únicas que ofrecen educación académica y técnica de las 152 instituciones educativas de carácter oficial en el municipio. Se manejó el protocolo para que los estudiantes respondieran de manera responsable y verídica la encuesta; esta fue dividida de acuerdo con los estudiantes de cada grupo; cada grupo se llevó por separado para explicarles el desarrollo y las condiciones y se les acompañó en el momento en que se les realizó la encuesta para poder aclarar sus dudas y evitar la filtración de información.

Se llevó a cabo el estudio de las condiciones físicas del plantel y la primera técnica de recolección de información fue la observación directa a través de diferentes visitas a los planteles educativos, durante las cuales se realizaron registros fotográficos, levantamiento de los panoramas de factores de riesgo, comparativos con normas y leyes vigentes en temas de salud, seguridad, higiene y medio ambiente; igualmente se realizaron diferentes entrevistas a docentes y directivos para luego comparar estas apreciaciones con la información obtenida en el estudio.

Para el análisis de las condiciones de las instituciones educativas se ha tomado el programa de Salud Ocupacional como herramienta y base de este estudio, por las características legales que tiene, y además por ser un programa en el cual actualmente se encuentran trabajando las instituciones educativas en las cuales se llevó a cabo la investigación. 
Esta clase de plan agrupa variedad de decretos y leyes referentes a la protección y prevención de la salud en todos sus aspectos, aunque legalmente el programa de Salud Ocupacional integra a la población trabajadora tal como lo dictan las leyes, entre ellas el Decreto 614 de 1984 [6] y la Resolución 1016 de 1989 [7], este programa promueve aspectos que se pueden enfocar hacia la población educativa de estas instituciones.

Para la elaboración de la encuesta, se tomaron en cuenta normas en salud ocupacional, usando estas como referentes para comparar las condiciones de higiene, seguridad, salud y medio ambiente, con la situación real de cada institución y el conocimiento que los estudiantes tienen sobre estos temas, además de cómo observan ellos su ambiente institucional.

El Código de Sanidad [8] en la sección de salud ocupacional habla de temas como requerimientos en las edificaciones que se puede complementar con normas técnicas [9] de arquitectura en zonas escolares, de las condiciones ambientales, físicas, químicas, biológicas de un lugar de trabajo además de la Seguridad Industrial, temas que son muy recurrentes en el espacio físico educativo. Entre otras normas que son guías para realizar el estudio, entre ellas: Norma Técnica Colombiana (NTC) 4595 - 4596 [10], Norma GTC 45 [11], Ley 100 de 1993 [12], Resolución 2400 de 1979 [13], Resolución 1016 de 1989 [14] y Decreto 1860 de 1994 - Proyecto Educativo Institucional (PEI) [15]

Para el análisis de los datos de las encuestas se procesó la información con la aplicación Microsoft Excel y así lograr precisión en los resultados y consignar los datos en gráficas de diferente tipo, con sus respectivos porcentajes y relación de datos.

\section{Resultados}

En las dos instituciones se encontró que los estudiantes con mayor frecuencia están matriculados en programas de Industria de la Madera, Electricidad, Informática y Metalmecánica, lo que indica la tendencia en la elección de programas industriales por el pensum académico que las dos instituciones les brindan a los estudiantes. En la figura 1 se puede observar lo descrito.

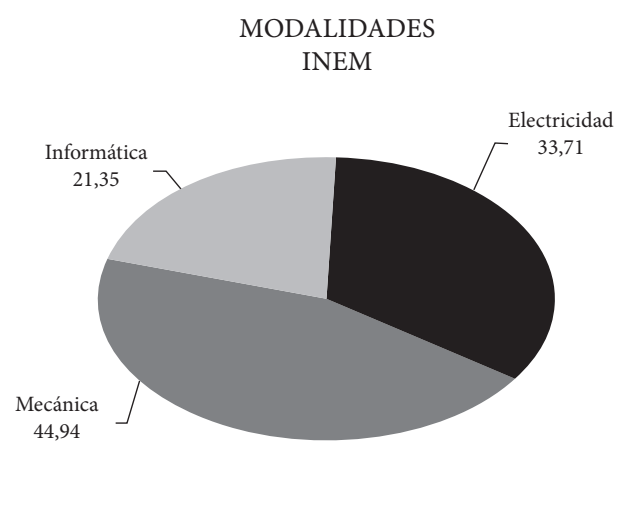

Figura 1. Frecuencia de estudiantes matriculados en modalidades industriales

Fuente: elaboración propia

\subsection{Servicios de salud y medicina preventiva}

De acuerdo con el artículo 127 de la Ley 9 de 1979 [8] es necesario que una institución educativa tenga un sitio y los recursos necesarios para prestar el servicio de primeros auxilios a los estudiantes, docentes y personal administrativo: se encontró que el colegio INEM además de tener enfermería, cuenta con el consultorio médico y odontológico, para brindar mejor atención a los estudiantes, pero tiene falencia en cuanto al registro médico que se debería llevar por cada alumno, en caso de existir algún incidente o accidente que permita conocer el historial de cada estudiante. La institución ITsim cuenta únicamente con enfermería y comité de bienestar. Esta información fue corroborada mediante la encuesta, ya que los estudiantes del colegio INEM conocen que existe servicio médico aunque no saben cuáles son exactamente sus servicios, mientras que los estudiantes del ITSIM aseguran no tener servicios de salud.

Se encontró que a un $80 \%$ de estudiantes del ITsim no se les realizó examen médico de ingreso; en el INEM este porcentaje es del 78\%, lo cual no es consecuente con lo que promueve un plan de salud ocupacional que en su primer subprograma que es medicina preventiva 
y del trabajo, propone que se realice un examen médico de ingreso y de egreso.

\subsection{Carné estudiantil-afiliación a riesgos}

Acerca de los seguros estudiantiles existe un gran contraste entre estas dos instituciones. Por una parte el colegio INEM, posee seguros y carné para el $99 \%$ de la población estudiantil. Esto se debe a que el costo de este carné está incluido dentro de la matrícula y es obligatorio. Para el caso del colegio Iтsim los estudiantes en un $20 \%$ lo han adquirido, esto se debe a que la compra del carné no es obligatoria y aunque su costo no es elevado, la mayoría de padres de familia han optado por no adquirirlo, quedando sin protección frente a riesgos derivados de sus actividades en los talleres y expuestos a los accidentes que en esta institución van desde lesiones leves, cortaduras, golpes, hasta amputaciones.

Sobre medicina preventiva se recolectó información de cuántas campañas o brigadas de salud se realizan en el año en estas instituciones educativas, y se encontró que no se llevan a cabo. En la entrevista realizada con las directivas informan que es difícil el manejo de ellas por el tiempo y el número de estudiantes, aunque han existido campañas, ofrecidas por instituciones de salud; en este aspecto se recomendó a las directivas, realizar más gestión con las empresas administradoras de riesgos profesionales, con las empresas promotoras de salud y en gran medida se recomendó hacer contactos con las facultades de las universidades de la región que tienen u ofrecen programas en el área de la salud, para que asílos estudiantes de estos colegios se beneficien de las campañas promovidas por estas entidades.

\section{Seguridad}

\subsection{Señalización}

Teniendo en cuenta el artículo 11 de la Resolución 1016 de 1989 [14], es necesario que una institución educativa en sus áreas académicas como industriales delimite o demarque las áreas de estudio, vías de circulación, salidas de emergencia, baños, ubicación de los extintores, zonas de evacuación, zonas de almacenamiento de basuras, zonas seguras, uso de elementos de protección personal y zonas peligrosas.

Con respecto a lo anterior se encontró gran deficiencia o mejor una alta ausencia de este tipo de señales, existen algunas y muy mal ubicadas, no hay conciencia ni en los estudiantes ni en las directivas de la importancia de estas señales y por ello no le prestan la atención debida a este punto, se recomendó crear conciencia de la importancia de la señalización a través de jornadas de sensibilización sobre este tema, es más, nosotros desde nuestro programa de ingeniería industrial podemos ayudar con estas sensibilizaciones, se está en conversaciones para trazar un plan de acción y desarrollar las sensibilizaciones anotadas anteriormente.

Es indispensable para los colegios contar con las siguientes señales de:

a. Obligación: es pertinente que en los talleres industria les, se implementen estas señales para que los estudiantes y docentes puedan observarlas y de acuerdo con su significado, exijan los elementos de protección requeridos para el desarrollo de sus actividades de aprendizaje.

b. Prevención: en los pasillos de los colegios donde se encuentran ubicadas las cajas de circuitos y en los talleres donde se presentan conexiones eléctricas, se deben implementar estas señales para que los estudiantes al observarlas eviten incidentes o accidentes.

c. Información: en los planteles educativos se deben ubicar estas señales para que los estudiantes, docentes y personal administrativo las visualicen en los pasillos y eviten posibles incidentes y accidentes.

\subsection{Capacitación sobre maquinaria}

Según la información adquirida por parte de los profesores e instructores de los talleres en las instituciones educativas, ellos brindan el conocimiento básico a los estudiantes sobre el funcionamiento de las máquinas, pero no tienen en cuenta las precauciones que los estudiantes deben tener para evitar accidentes. A pesar de esto, los estudiantes se sienten satisfechos por el conocimiento adquirido para poder desempeñarse en su trabajo acádemico.

\subsection{Elementos de protección personal}

Los elementos de protección personal tienen como función proteger las diferentes partes del cuerpo, para evitar que las personas se encuentren expuestas a factores de riesgo. En las instituciones educativas, los elementos de protección son escasos en especial en los laboratorios, lo que dificulta el desarrollo del aprendizaje para los estudiantes. En la tabla 1 se presentan los elementos de protección personal que deberían utilizar los estudiantes para su aprendizaje. De igual forma, los estudiantes han tenido algunos accidentes por la manipulación de maquinaria y herramienta industrial sin los elementos de protección personal e imprudencia de los estudiantes. 
Tabla 1. Elementos de protección personal que deberían utilizar los estudiantes para su aprendizaje

\begin{tabular}{|c|c|c|c|}
\hline EPP & INDICACIÓN & HALLAZGO & NORMA \\
\hline \multirow{4}{*}{ CASCO } & \multirow{2}{*}{$\begin{array}{l}\text { En los talleres de Metalmecánica y Ebanistería } \\
\text { (Industria de la Madera) se requiere el uso } \\
\text { obligatorio de casco de seguridad, para la } \\
\text { protección de la cabeza contra impactos leves de } \\
\text { objetos en caída libre y golpes contra objetos fijos. }\end{array}$} & \multirow{2}{*}{$\begin{array}{l}\text { Se encontró que la cantidad de } \\
\text { cascos que se tiene, no corresponde } \\
\text { al número necesario para todos } \\
\text { los estudiantes, además no están } \\
\text { en buenas condiciones los cascos } \\
\text { existentes, la mayoría de ellos tiene } \\
\text { los tafiletes en mal estado. }\end{array}$} & ANSI z89.1 Protective Helmet \\
\hline & & & NTC 1523 Cascos de Seguridad \\
\hline & \multirow{2}{*}{$\begin{array}{l}\text { En el taller de Electricidad se requiere el uso } \\
\text { obligatorio de cascos de seguridad y de casco de } \\
\text { seguridad dieléctrico, para la protección de la } \\
\text { cabeza contra choques eléctricos y radiación solar. }\end{array}$} & \multirow{2}{*}{$\begin{array}{l}\text { Al igual que en el caso anterior, los } \\
\text { cascos no están en buen estado, y } \\
\text { además los cascos dieléctricos son } \\
\text { muy pocos. }\end{array}$} & $\begin{array}{l}\text { ANSI z89.1 - } 2003 \text { "For Industrial } \\
\text { Head Protection". }\end{array}$ \\
\hline & & & NTC 1523 Cascos de Seguridad \\
\hline $\begin{array}{l}\text { PROTECCIÓN } \\
\text { AUDITIVA }\end{array}$ & $\begin{array}{l}\text { En los talleres de Metalmecánica y Ebanistería } \\
\text { se requiere el uso obligatorio de protectores } \\
\text { auditivos tipo copa, que permiten aislar y atenuar } \\
\text { los niveles de ruido hasta } 29 \mathrm{~dB} \text { que puedan ser } \\
\text { perjudiciales para la salud, sin afectar la audición } \\
\text { en la conversación normal. }\end{array}$ & $\begin{array}{l}\text { En cuanto a la existencia y uso de } \\
\text { protectores auditivos se encontró } \\
\text { que sí hay cantidad suficiente y } \\
\text { sí los utilizan los estudiantes, se } \\
\text { recomienda mantener el inventario } \\
\text { de protectores auditivos. }\end{array}$ & ANSI s3. 19-1974 \\
\hline \multirow{2}{*}{$\begin{array}{l}\text { PROTECCIÓN } \\
\text { OCULAR }\end{array}$} & $\begin{array}{l}\text { Para los talleres de Electricidad, Ebanistería y } \\
\text { Metalmecánica es necesario que los estudiantes } \\
\text { utilicen anteojos de seguridad (gafas de } \\
\text { seguridad), con resistencia a impactos de baja } \\
\text { energía, partículas de polvo y partículas finas. }\end{array}$ & $\begin{array}{l}\text { En cuanto a protección ocular } \\
\text { se encontró que no hay cantidad } \\
\text { suficiente de gafas para protección. }\end{array}$ & ANSI Z-87.1 \\
\hline & $\begin{array}{l}\text { Para los laboratorios de Física y Química se } \\
\text { requiere el uso de anteojos de seguridad, con } \\
\text { resistencia a líquidos, partículas de polvo, } \\
\text { partículas finas y gases. }\end{array}$ & $\begin{array}{l}\text { En cuanto a protección ocular } \\
\text { se encontró que no hay cantidad } \\
\text { suficiente de gafas para protección. }\end{array}$ & ANSI Z-87.1 \\
\hline \multirow{5}{*}{$\begin{array}{l}\text { PROTECCIÓN } \\
\text { FACIAL }\end{array}$} & \multirow{2}{*}{$\begin{array}{l}\text { Para el taller de Metalmecánica se requiere el uso } \\
\text { de la careta para esmerilar con sistema de ajuste } \\
\text { Ratchet, para la protección de los ojos y cara de } \\
\text { polvo, viruta y chispas. }\end{array}$} & \multirow[t]{2}{*}{$\begin{array}{l}\text { Se encontró que se tienen suficientes } \\
\text { caretas para esmerilar y se } \\
\text { encuentran en buen estado. }\end{array}$} & $\begin{array}{l}\text { ANSI z87.1 "Practice for } \\
\text { Occupational and educational eye } \\
\text { and face protection" }\end{array}$ \\
\hline & & & $\begin{array}{l}\text { NTC } 3610 \text { "Caretas para soldar y } \\
\text { protección facial". }\end{array}$ \\
\hline & \multirow{2}{*}{$\begin{array}{l}\text { Para el taller de Metalmecánica se requiere el uso } \\
\text { de la careta para soldar, para proteger los ojos y } \\
\text { la cara de trabajos donde se emplea soldadura de } \\
\text { arco. }\end{array}$} & \multirow[t]{2}{*}{$\begin{array}{l}\text { Se encontró que se tienen suficientes } \\
\text { caretas para soldar y se encuentran } \\
\text { en buen estado. }\end{array}$} & $\begin{array}{l}\text { ANSI z87.1 "Practice for } \\
\text { Occupational and educational eye } \\
\text { and face protection" }\end{array}$ \\
\hline & & & $\begin{array}{l}\text { NTC } 3610 \text { "Caretas para soldar y } \\
\text { protección facial". }\end{array}$ \\
\hline & $\begin{array}{l}\text { Para el taller de Ebanistería se requiere el uso } \\
\text { de la careta multipropósito con visor en acetato, } \\
\text { recomendada para proteger los ojos y la cara de } \\
\text { partículas emitidas por trabajos como pulido, } \\
\text { tallado y cepillado de la madera. }\end{array}$ & $\begin{array}{l}\text { Se encontró que se tienen suficientes } \\
\text { caretas multipropósito y se } \\
\text { encuentran en buen estado. }\end{array}$ & $\begin{array}{l}\text { NTC } 3610 \text { "Caretas para soldar y } \\
\text { protección facial". }\end{array}$ \\
\hline \multirow[t]{2}{*}{$\begin{array}{l}\text { GUANTES DE } \\
\text { SEGURIDAD }\end{array}$} & $\begin{array}{l}\text { Para el taller de Metalmecánica se requiere el uso } \\
\text { de guantes para soldador, para la protección de las } \\
\text { manos del calor radiante y directo indeterminista. }\end{array}$ & $\begin{array}{l}\text { Se encontró que se tienen suficientes } \\
\text { guantes y están en buen estado. }\end{array}$ & $\begin{array}{l}\text { NTC } 2190 \text { "Guantes de seguridad } \\
\text { para uso industrial fabricados en } \\
\text { carnaza y cuero" }\end{array}$ \\
\hline & $\begin{array}{l}\text { Para el taller de Ebanistería se requiere el uso de } \\
\text { guantes para la protección de las manos. }\end{array}$ & $\begin{array}{l}\text { Se encontró que se tienen suficientes } \\
\text { guantes y están en buen estado. }\end{array}$ & $\begin{array}{l}\text { NTC } 2190 \text { "Guantes de seguridad } \\
\text { para uso industrial fabricados en } \\
\text { carnaza y cuero" }\end{array}$ \\
\hline $\begin{array}{l}\text { PROTECCIÓN } \\
\text { RESPIRATORIA }\end{array}$ & $\begin{array}{l}\text { Para el taller de Metalmecánica se requiere } \\
\text { el uso de mascarilla, para la protección de las } \\
\text { vías respiratorias de polvo metálico y humos } \\
\text { generados por soldadura. }\end{array}$ & $\begin{array}{l}\text { Se encontraron suficientes } \\
\text { mascarillas para protección de vías } \\
\text { aéreas y están en buen estado. }\end{array}$ & $\begin{array}{l}\text { NTC } 1584 \text { "Equipos de protección } \\
\text { respiratoria, definiciones y } \\
\text { clasificación" } \\
\text { NTC } 1733 \text { "Equipos de protección } \\
\text { respiratoria. }\end{array}$ \\
\hline
\end{tabular}

Fuente: elaboración propia con base en fichas técnicas [16] 


\subsection{Extintores}

Por medio de visitas realizadas a los colegios, se pudo observar que la señalización en cuanto a extintores es escasa e inadecuada, de igual manera la ubicación no es la correcta, esto puede causar distracción en los estudiantes y docentes en caso de cualquier eventualidad. A continuación, en la tabla 2, se presenta la lista de verificación de extintores de los colegios en estudio.

Tabla 2. Lista de verificación de extintores de los institutos INEM e ITSIM

\begin{tabular}{|c|c|c|c|c|c|}
\hline \multirow{3}{*}{ LUGAR DE TRABAJO } & \multicolumn{4}{|c|}{$\begin{array}{l}\text { INSTITUTO TÉCNICO SUPERIOR INDUSTRIAL MUNICIPAL - ITSIM } \\
\text { INSTITUCIÓN EDUCATIVA LUIS DELFÍN INSUATY RODRÍGUEZ - INEM } \\
\text { INSPECCIONES DE EXTINTORES }\end{array}$} & \multirow[b]{2}{*}{ AA } \\
\hline & \multirow{2}{*}{ Institución Educativa INEM } & \multirow{2}{*}{ FECHA } & DD & MM & \\
\hline & & & 1 & Marzo & 2011 \\
\hline \multicolumn{6}{|c|}{ El encargado de la inspección determina el estado del extintor según las siguientes indicaciones: } \\
\hline \multicolumn{2}{|c|}{$\begin{array}{l}\text { Marca con una X en el recuadro, según lo observado en la } \\
\text { inspección }\end{array}$} & DEFICIENTE & REGULAR & BUENO(A) & NO APLICA \\
\hline \multicolumn{2}{|c|}{ PARTES O ELEMENTOS A INSPECCIONAR } & $\mathrm{D}$ & $\mathbf{R}$ & B & N/A \\
\hline \multicolumn{2}{|c|}{ ALTURA DEL PISO (RECOMENDADA $1,30 \mathrm{~m}$ ) } & $\mathrm{X}$ & & & \\
\hline \multicolumn{2}{|l|}{ BOQUILLA } & & $\mathrm{X}$ & & \\
\hline \multicolumn{2}{|c|}{ CAPACIDAD DEL EXTINTOR } & & & $\mathrm{X}$ & \\
\hline \multicolumn{2}{|l|}{ CILINDRO } & & & $\mathrm{X}$ & \\
\hline \multicolumn{2}{|l|}{ DEMARCACIÓN DEL PISO } & $\mathrm{X}$ & & & \\
\hline \multicolumn{2}{|l|}{ MANGUERA } & $\mathrm{X}$ & & & \\
\hline \multicolumn{2}{|l|}{ MANIJA DE DESCARGA } & $\mathrm{X}$ & & & \\
\hline \multicolumn{2}{|l|}{ MANÓMETRO } & $\mathrm{X}$ & & & \\
\hline \multicolumn{2}{|l|}{ PASADOR } & $\mathrm{X}$ & & & \\
\hline \multicolumn{2}{|l|}{ PRESIÓN } & $\mathrm{X}$ & & & \\
\hline \multicolumn{2}{|l|}{ SELLO DE SEGURIDAD } & & & $\mathrm{X}$ & \\
\hline \multicolumn{2}{|l|}{ SEÑALIZACIÓN } & & $\mathrm{X}$ & & \\
\hline \multicolumn{2}{|c|}{ SEÑALIZACIÓN LUMINOSA } & $\mathrm{X}$ & & & \\
\hline \multicolumn{2}{|l|}{ TIPO DE EXTINTOR } & & & $\mathrm{X}$ & \\
\hline \multicolumn{2}{|l|}{ UBICACIÓN } & $\mathrm{X}$ & & & \\
\hline \multicolumn{6}{|c|}{ OBSERVACIONES } \\
\hline
\end{tabular}

Fuente: elaboración propia

\subsection{Brigada de emergencia}

Los estudiantes de las instituciones educativas manifestaron que no tienen conocimiento, ni han recibido ninguna información sobre brigadas de emergencia. Según las directivas de los colegios el plan de emergencias sí está diseñado, pero ha sido complicado implementar dichos planes de emergencia, es lo que expresan las directivas, se recomienda cuanto antes implementar y socializar dichos planes de emergencia.

Las dos instituciones se encuentran expuestas a riesgos naturales inminentes a raíz de dos factores naturales como son: a. El Volcán Galeras de la ciudad de Pasto es el primer factor de riesgo al que estas instituciones se encuentran expuestas. La ubicación de ellas es una zona de riesgo determinado por IngEominas [17] donde la amenaza puede ser por movimientos sísmicos como también por emisión de gases, cenizas, y flujos piroclastos.

b. La Quebrada Mijitayo es el segundo factor natural al que se encuentran expuestos. Esta quebrada sube de nivel en épocas de lluvia ocasionando que el agua baje por zonas residenciales y por las zonas de tránsito de las instituciones, exponiendo a los estudiantes y habitantes en general a posibles incidentes y accidentes. 


\subsection{Higiene y medio ambiente}

Puesto que la higiene contribuye con la reducción y eliminación de factores de riesgo a los cuales están expuestos los estudiantes y al cuidado del medio ambiente, además de estar relacionado con el aseo e higiene de manera directa, con el fin de determinar el cuidado y manejo de estos temas se realizó un estudio basado en los siguientes puntos: existencia de aseo en el sitio de trabajo y aseo en los baños.

Los resultados que se obtuvieron se presentan en la figura 2.

\section{PREGUNTAS HIGIENE Y MEDIO AMBIENTE INEM}

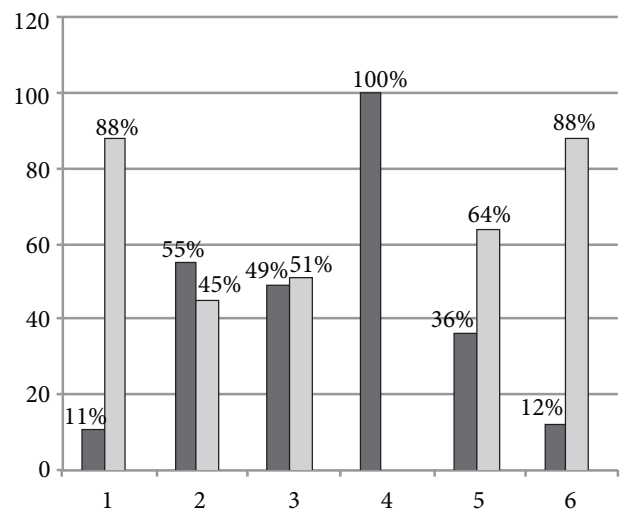

Figura 2. Higiene y medio ambiente Fuente: elaboración propia

La información recolectada se ha resumido en porcentajes basados en la observación, entrevistas y la encuesta a los estudiantes, en este caso se verificó que en la ITsim se hace el aseo cada 3 horas, hay 5 basureros por área o bloque de estudio y hay campañas para mejorar las condiciones de aseo de la institución, además de que en áreas industriales el aseo lo realiza cada alumno tal y como lo manifestaron las directivas, docentes y estudiantes, en cuanto a esto las instituciones educativas se preocupan por que sus alumnos se concienticen y ayuden a mejorar el medio ambiente.

Sobre higiene personal los baños son escasos en la iтsim para el número total de alumnos en la institución existen 20 servicios en excelentes condiciones, para las áreas industriales los baños están en su mayoría fuera de uso.

Buscando responder a los signos de los tiempos de hoy, las instituciones se han preocupado por concientizar a sus estudiantes sobre la necesidad del cuidado del medio ambiente. Es por ello que la comunidad estudiantil se hace conocedora del tema. Pero existe una gran discre-

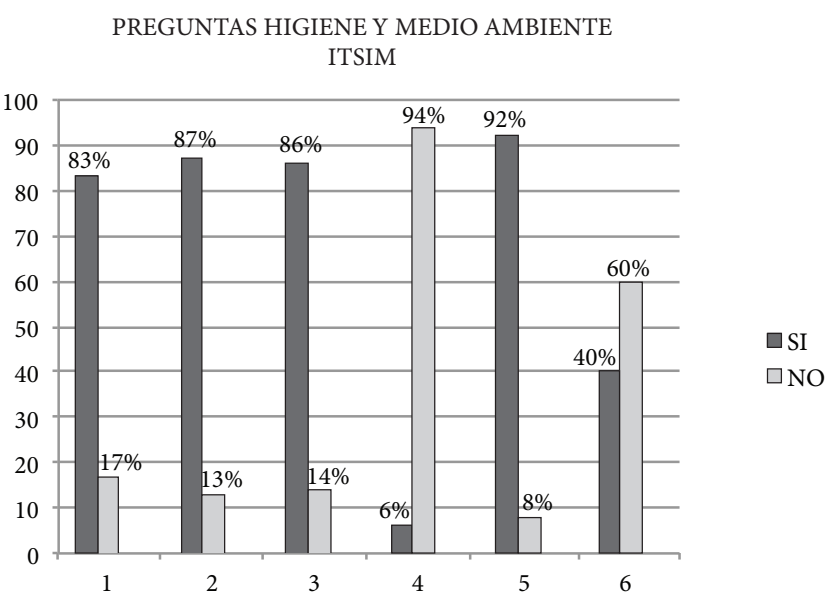

pancia en cuanto al espacio de las zonas verdes, en donde el ITSIM por su ubicación física no cuenta con estas.

En la institución INEM, la situación es totalmente diferente, ya que cuenta con muchas zonas verdes; en cuanto al aseo, aunque posee basureros, estos son insuficientes; en cambio, en esta institución, cada bloque posee baños para hombres y mujeres que son suficientes para el número total de estudiantes, aunque la mayoría de estos estén en condiciones regulares.

Las brigadas ambientales y de aseo son un problema para las institución INEM, esto corroborado con información suministrada por directivas, ya que ellos relacionan el hecho de tener más de seis mil (6000) [10] estudiantes entre la jornada de la mañana y la tarde con la coordinación de brigadas ambientales o de cualquier otro tipo. Las brigadas de aseo sí han sido realizadas en menor medida; además de esto, el control de basuras se dificulta por ser un plantel de gran espacio físico. En la tabla 3, se relaciona el estado de las instalaciones según las normas y leyes establecidas. 
Tabla 3. Estado de las instalaciones según normas y leyes

\begin{tabular}{|c|c|c|}
\hline & Hallazgo & Norma de referencia \\
\hline 1 & $\begin{array}{l}\text { En algunos salones de clase de los pisos superiores del edificio, los ventanales no } \\
\text { tienen instaladas rejillas de seguridad que prevengan la caída de estudiantes que } \\
\text { asumen comportamientos de riesgo. }\end{array}$ & $\begin{array}{l}\text { NTC } 9545 \text { (Ingeniería civil y arquitectura para ambientes } \\
\text { escolares) } \\
\text { Resolución número } 02400 \text { de mayo } 22 \text { de } 1979 \\
\text { (Disposiciones sobre vivienda, higiene y seguridad } \\
\text { industrial en establecimientos de trabajo) }\end{array}$ \\
\hline 2 & $\begin{array}{l}\text { En el plantel educativo se observaron instalaciones eléctricas deficientes y de mal } \\
\text { aspecto. Por otra parte, algunas deficiencias eléctricas implican deficiencias en la } \\
\text { iluminación de los espacios de trabajo y de estudio al carecer de luminarias. }\end{array}$ & $\begin{array}{l}\text { Ley } 9 \text { de } 1979 \text { sobre riesgos eléctricos } \\
\text { NTC } 2050 \text { (Código Eléctrico Colombiano) } \\
\text { NTC } 9545 \text { (Ingeniería civil y arquitectura para ambientes } \\
\text { escolares) }\end{array}$ \\
\hline 3 & $\begin{array}{l}\text { En la institución educativa se observó que en las aulas de clases las lámparas } \\
\text { presentan conexiones inadecuadas además de no estar sujetas. Se puede } \\
\text { considerar la existencia de un riesgo de seguridad al estar los estudiantes } \\
\text { expuestos a esto. } \\
\text { Por otra parte, los techos son de madera y las conexiones eléctricas de } \\
\text { las lámparas no tienen la debida aislación, por lo cual puede generar un } \\
\text { cortocircuito. }\end{array}$ & $\begin{array}{l}\text { Ley } 9 \text { de } 1979 \text { de las condiciones ambientales, muros y } \\
\text { techos, Iluminación (arts. } 99 \text {, 195, } 196 \text { y 197) } \\
\text { Resolución número } 02400 \text { de mayo } 22 \text { de } 1979 \text { sobre la } \\
\text { iluminación } \\
\text { NTC } 9545 \text { (Ingeniería civil y arquitectura para ambientes } \\
\text { escolares) }\end{array}$ \\
\hline 4 & $\begin{array}{l}\text { En los pasillos de las instituciones se encontró que la mayoría de las cajas de } \\
\text { circuitos no presentan la debida seguridad, estos pueden ser causantes de riesgos } \\
\text { eléctricos por contacto directo. }\end{array}$ & $\begin{array}{l}\text { Ley } 9 \text { de } 1979 \text { sobre las condiciones ambientales (art. } \\
\text { 99) } \\
\text { Resolución número } 02400 \text { de mayo } 22 \text { de } 1979 \text { sobre la } \\
\text { Prevención }\end{array}$ \\
\hline 5 & $\begin{array}{l}\text { Por la ubicación geográfica del colegio, este se encuentra expuesto a las amenazas } \\
\text { naturales como son el Volcán Galeras y la Quebrada de Mijitayo. }\end{array}$ & $\begin{array}{l}\text { Ley } 9 \text { de } 1979 \text { (art. 96) } \\
\text { Resolución } 1016 \text { de } 1989 \\
\text { Decreto de Ley } 1295 \text { de } 1994\end{array}$ \\
\hline 6 & $\begin{array}{l}\text { Los baños en el plantel educativo presentan deterioro, desaseo y en muchos } \\
\text { casos su funcionamiento es totalmente inhabilitado, esto generando condiciones } \\
\text { peligrosas para los estudiantes, relacionados con los riesgos biológicos. }\end{array}$ & $\begin{array}{l}\text { Ley } 9 \text { de } 1979 \text { (arts. } 179 \text { - 212) } \\
\text { Resolución } 2400 \text { de } 1979 \text { (arts. 20, } 21 \text { y 22) }\end{array}$ \\
\hline 7 & $\begin{array}{l}\text { Algunos baños en la institución no tienen la adecuada ventilación, generando } \\
\text { náuseas, alergias, irritación de los ojos y tos, estos son relacionados con los } \\
\text { riesgos biológicos. }\end{array}$ & $\begin{array}{l}\text { Resolución } 2400 \text { de } 1979 \text { (arts. 20, } 21 \text { y 22) } \\
\text { Ley } 9 \text { de } 1979 \text { (art. 109) } \\
\text { NTC } 5183 \text { (Ventilación para una calidad aceptable del } \\
\text { aire en espacios interiores) }\end{array}$ \\
\hline 8 & $\begin{array}{l}\text { Algunos baños son utilizados como bodega, esto puede causar un accidente a los } \\
\text { estudiantes y de igual manera causa una mala impresión. }\end{array}$ & Ley 9 de 1979 \\
\hline 9 & $\begin{array}{l}\text { Las conexiones eléctricas no son las adecuadas en los diferentes talleres, estas } \\
\text { pueden causar riesgo eléctrico por contacto directo, de igual manera puede causar } \\
\text { cortos circuitos. }\end{array}$ & $\begin{array}{l}\text { NTC } 2050 \text { (Código Eléctrico Colombiano) } \\
\text { RETIE } \\
\text { Ley } 9 \text { de } 1979 \text { sobre las condiciones ambientales (art. 99) } \\
\text { Resolución número } 02400 \text { de mayo } 22 \text { de } 1979 \text { sobre el } \\
\text { Riesgo Eléctrico }\end{array}$ \\
\hline 10 & $\begin{array}{l}\text { En el colegio se encontró sobrecarga en los tomacorriente, esto puede generar } \\
\text { riesgo eléctrico y causar accidentes a los estudiantes y daños en las máquinas e } \\
\text { infraestructura. }\end{array}$ & $\begin{array}{l}\text { NTC } 2050 \text { (Código Eléctrico Colombiano) } \\
\text { RETIE } \\
\text { Ley } 9 \text { de } 1979 \text { sobre las condiciones ambientales (art. } \\
\text { 99). } \\
\text { Resolución número } 02400 \text { de mayo } 22 \text { de } 1979 \text { sobre el } \\
\text { Riesgo Eléctrico }\end{array}$ \\
\hline 11 & $\begin{array}{l}\text { En los pupitres donde los estudiantes realizan sus prácticas en el área de } \\
\text { electricidad se encontró que están deteriorados y en mal estado, riesgo eléctrico } \\
\text { para los estudiantes por contacto directo. }\end{array}$ & $\begin{array}{l}\text { NTC } 1819 \\
\text { NTC } 1943\end{array}$ \\
\hline 12 & $\begin{array}{l}\text { Los estudiantes al realizar sus actividades industriales no utilizan los elementos de } \\
\text { protección personal. }\end{array}$ & $\begin{array}{l}\text { Ley } 9 \text { de } 1979 \text { (Maquinaria, equipos, herramienta y } \\
\text { elementos de protección personal; arts. 112-124) }\end{array}$ \\
\hline 13 & $\begin{array}{l}\text { La mala postura de los estudiantes al realizar sus actividades industriales en las } \\
\text { dos instituciones no es la correcta, lo cual puede causar enfermedades a largo } \\
\text { plazo como consecuencia de un riesgo ergonómico. }\end{array}$ & $\begin{array}{l}\text { Decreto } 1295 \text { de } 1994 \\
\text { Ley } 9 \text { de } 1979 \\
\text { NTC } 9545 \text { (Ingeniería civil y arquitectura para ambientes } \\
\text { escolares) } \\
\text { Decreto } 1832 \text { de agosto } 3 \text { de } 1994 \text { (el cual adopta la } \\
\text { tabla de enfermedades profesionales) }\end{array}$ \\
\hline
\end{tabular}




\begin{tabular}{|c|c|c|}
\hline & Hallazgo & Norma de referencia \\
\hline 14 & $\begin{array}{l}\text { Se encontró que en la mayoría de gradas que existen en las instituciones } \\
\text { educativas no tienen la adecuada señalización. }\end{array}$ & $\begin{array}{l}\text { Ley } 9 \text { de } 1979 \\
\text { NTC } 9545 \text { (Ingeniería Civil y Arquitectura para } \\
\text { ambientes escolares) } \\
\text { Resolución } 2400 \text { de } 1979 \\
\text { ICONTEC } \\
\text { NTC } 1461\end{array}$ \\
\hline 15 & La ubicación y la señalización de los extintores no es la correcta en el colegio. & $\begin{array}{l}\text { Ley } 9 \text { de } 1979 \\
\text { NTC } 9545 \text { (Ingeniería civil y arquitectura para ambientes } \\
\text { escolares) } \\
\text { Resolución } 2400 \text { de } 1979 \\
\text { Norma } 101 \text { de la NFPA (Código de Seguridad Humana) }\end{array}$ \\
\hline 16 & $\begin{array}{l}\text { En la institución educativa se observó la falta de preocupación por el aseo y la } \\
\text { separación de las basuras. } \\
\text { Estas condiciones generan condiciones de riego peligrosas asociadas a riesgos } \\
\text { biológicos. }\end{array}$ & $\begin{array}{l}\text { Resolución número } 02400 \text { de mayo } 22 \text { de } 1979 \text { sobre el } \\
\text { orden y limpieza } \\
\text { Ley } 9 \text { de } 1979 \text { (arts. 23, } 24 \text { y 25) }\end{array}$ \\
\hline
\end{tabular}

Fuente: elaboración propia

\section{Discusión}

La educación es una etapa por la cual un individuo debe pasar desde su niñez hasta la adultez. Si el ámbito y negocio educativo en sí es tan amplio, este debe tener todos los parámetros necesarios para poder ofrecer un servicio adecuado. Un estudiante pasa muchas horas en un plantel académico. Por lo tanto, el lugar en donde se encuentra debe permitir el desarrollo correcto del aprendizaje sin ninguna dificultad.

Las normas a las cuales se acoge una institución educativa deben ser más exigentes, ya que una institución maneja un porcentaje amplio de personas todos los días. Debe ser responsabilidad del Estado procurar condiciones de bienestar a los alumnos y personas en general.

La prevención en este caso no puede ser opcional, ya que un padre de familia no sólo está pagando por educar a su hijo, sino que él sabe que un plantel es un segundo hogar desde que ingresa hasta que sale; por lo tanto, pone la confianza en la institución en la que no se expondrán a sus hijos a riesgos ni a posibles accidentes.

Es necesario trabajar por que la población estudiantil, que es grande, tenga condiciones adecuadas y se puedan implementar normas que ayuden y beneficien a esta. Por ejemplo, ser más rígidos en cuanto hacer obligatorios lo comités de higiene y seguridad industrial, en este caso en el lugar de estudio [14].

$\mathrm{Al}$ hacer el anterior estudio en las instituciones educativas, se observó que en muchos casos hay intenciones, deseos de mejora y aplicación de una normatividad en cuanto a la implementación de programas de salud ocupacional. Pero la falta de conocimiento en estas temáticas no permite que se desarrollen estas ideas. Además de observar que las directivas relacionan el programa de salud ocupacional como afiliaciones a ARP y enfermedad profesional, pero no como un programa que contiene salud, seguridad, higiene y medio ambiente. Aunque el Ministerio de Educación tiene guías que pueden ser aplicadas en las diferentes instituciones educativas, es importante determinar en qué condiciones se encuentra cada plantel y poder así solventar sus necesidades.

Generalizar no es beneficio, ya que no todos los planteles educativos no poseen los mismos problemas ni se encuentran en iguales condiciones. Por lo tanto, es necesario gestionar un programa completo de salud ocupacional en una institución para evitar riesgos, enfermedades, catástrofes, y así mejorar la calidad de la educación.

\section{Conclusiones y recomendaciones}

Términos como seguridad, higiene, salud se emplean cuando se habla de la protección de la salud y de la seguridad de las personas que hacen parte de un entorno laboral, dando por hecho que son esas personas quienes deben encargarse de su seguridad; si bien eso en parte es cierto, también hay que tener en cuenta que son los directivos de esos entornos laborales quienes deben velar por la prevención de cualquier incidente que afecte la salud y la seguridad de las personas, para el caso de esta investigación, el entorno laboral se asocia con los espacios académicos que las instituciones técnicas educativas disponen para que los estudiantes lleven a cabo su aprendizaje y por ello toma alta relevancia la sensibilización en temas de seguridad industrial, por ejemplo, 
para que no se presenten accidentes que pueden ser leves o en el peor de los casos fatales.

Ahora bien, se pretende fomentar una cultura de prevención en las instituciones técnicas educativas, sensibilizando a la comunidad educativa sobre la importancia de la prevención y lo que es más importante, sobre el cuidado personal que cada uno de ellos debe tener para que no le ocurra ningún incidente o ningún accidente de trabajo; no es suficiente hacer o implementar un programa de salud ocupacional, o imponer qué se debe o tiene que cumplir con esta o aquella ley que rige en el momento; no, lo importante es la concientización de los peligros y riesgos a los cuales se puede exponer un estudiante o un docente en uno de los espacios académicos en los cuales se desarrollan las actividades académicas en las instituciones técnicas educativas objeto de esta investigación.

Los administrativos de estas instituciones técnicas educativas deben estar abiertos a las recomendaciones que salgan de los planes de acción generados a partir de la identificación de los riesgos en los panoramas de factores de riesgo levantados por expertos y con base en la normatividad y legislación vigente para ello; no únicamente en lo que tiene que ver con los aspectos económicos encaminados a realizar las inversiones necesarias para mejoras locativas o para la adquisición de elementos de protección personal; es decir, no sólo para cuestiones de inversión de dinero, sino que deben también estar dispuestos a participar activamente de todas las actividades que se programen para la prevención de cualquier incidente que resulte lamentable.

Los estudiantes, como principal agente expuesto al peligro, deben ser conscientes de su vulnerabilidad y, por ende, no sólo participar como invitados de piedra en la sensibilización sobre riesgos, sino que deben ser ellos los artífices de una cultura de prevención muy fuerte que ayude de manera significativa a las directivas de las instituciones técnicas educativas a mejorar día a día esa cultura de la prevención, para beneficio propio y de cualquier persona que, de pronto, esté expuesta a los riesgos identificados previamente en los lugares de trabajo que pueden presentar riesgos.

Se recomienda de manera especial a las directivas de las instituciones técnicas educativas crear o aumentar el acercamiento con la administradora de riesgos laborales (ARL) en la cual se encuentren afiliados, ya que de acuerdo con las disposiciones legales vigentes en Colombia, estas están en la obligación de prestar gratuitamente toda la capacitación en prevención que sea necesaria con el fin de minimizar al máximo cualquier presencia de riesgo que pueda llevar a algún accidente no deseado con consecuencias fatales.

\section{Agradecimientos}

El autor agradece muy especialmente a las directivas de los colegios que participaron en esta investigación y también a los estudiantes de octavo semestre del programa de Ingeniería Industrial de la Universidad Cooperativa de Colombia Pasto que cursaron la asignatura Salud Ocupacional, en especial, a Alejandra Rodríguez y Diana Calvache, por su excelente colaboración.

\section{Referencias}

[1] Departamento Administrativo Nacional de Estadística [DAne], Metodología Proyecciones de Población y Estudios Demográficos. Bogotá: Dirección de Difusión, Mercadeo y Cultura Estadística, 2009. [En línea]. Disponible en: http://www.dane.gov.co/files/investigaciones/fichas/Proyecciones_poblacion.pdf

[2] M. R. Spiegel, Probabilidad y estadística (Schaum). México: McGraw Hill, 2010.

[3] G. C. Canavos, Probabilidad y estadística: aplicaciones y métodos. México: McGraw Hill, 1990.

[4] Institución Educativa InEM de Pasto. 2011. [En línea]. http://www.inempasto.edu.co

[5] Institución Educativa ITsim de Pasto. 2011 [Online]. Disponible en: http://www.tecnicoindustrialpasto.edu.co

[6] Presidencia de la República de Colombia. (1984, Marzo 14). Decreto de 614 de 1984 por el cual se determinan las bases para la organización y administración de Salud Ocupacional en el país. [En línea]. Disponible en: http:// www.alcaldiabogota.gov.co/ sisjur/normas/Norma1. jsp?i=1357

[7] Ministerio de Trabajo y Ministerio de Seguridad Social y de Salud. (1989, Marzo 31). Resolución 1016 de 1989. [En línea]. Disponible en: http://www.alcaldiabogota. gov.co/sisjur/normas/Norma1.jsp? $\mathrm{i}=5412$

[8] Congreso de Colombia. (1979, Enero 24). Ley 9 de 1979, por la cual se dictan medidas sanitarias. [En línea]. Disponible en: http://www.alcaldiabogota.gov.co/ sisjur/ normas/Norma1.jsp?i=1177

[9] Ministerio de Educación Nacional. (2006, Marzo). Normas Técnicas Colombianas NTC 4595 y NTC 4596. Ingeniería Civil y Arquitectura. Planeamiento y Diseño de Instalaciones y Ambientes Escolares. [En línea]. Disponible en: http://www.mineducacio.gov.co/1621/articles-96894_Archivo_pdf.pdf 
[10] Instituto Colombiano de Normas Técnicas y Certificación, ICONTEC. Compendio Sistema de gestion en seguridad y salud ocupacional. Bogotá: ICONTEC, 2012.

[11] Instituto Colombiano de Normas Técnicas y Certificación, ICONTEc. Guía Técnica Colombiana gTC 45. Guía para la identificación de los peligros y la valoración de los riesgos en seguridad y salud ocupacional. Bogotá: ICONTEC, 2010.

[12] Congreso de la República. (1993, Diciembre 23). Ley 100 de 1993, por la cual se crea el sistema de seguridad social integral y se dictan otras disposiciones. [En línea]. Disponible en: http://www.alcaldiabogota.gov.co/sisjur/normas/ Norma1.jsp?i=5248

[13] Ministerio de Trabajo y Seguridad Social. (1979, Mayo 22). Resolución 2400 de 1979, por la cual se establecen algunas disposiciones sobre vivienda, higiene y seguridad en los establecimientos de trabajo. [En línea]. Disponible en: http://copaso.upbbga.edu.co/legislacion/Res.24001979.pdf

[14] Ministerio de Trabajo y Ministerio de Seguridad Social y de Salud. (1989, Marzo 31). Resolución 1016 del 1989, por la cual se reglamenta la organización, funcionamiento y forma de los Programas de Salud Ocupacional que deben desarrollar los patronos o empleadores en el país. [En línea]. Disponible en: http://www.alcaldiabogota. gov.co/sisjur/normas/Norma1.jsp?i=5412

[15] Ministerio de Educacion Nacional. (1994, Agosto 3). Decreto 1860 de 1994, Por el cual se reglamenta parcialmente la ley 115 de 1994, en los aspectos pedagógicos y organizativos generales. [En línea]. Disponible en: http:// www.mineducacion.gov.co/1621/articles-172061_archivo_pdf_decreto1860_94.pdf

[16] ARSEG. Primero el hombre. Fichas técnicas. [Online]. Disponibles en: http://www.arseg.com.co/productos/ fichas-tecnicas

[17] Instituto Colombiano de Geiología y Minería, INGEOMINAS. (1997). Mapa de Amenaza Volcánica de Galeras. [En línea]. Disponible en: http://intranet.ingeominas. gov.co/pasto/Mapa_de_Amenaza_Volc\%C3\%A1nica_ de_Galeras_-_Tercera_Versi\%C3\%B3n 